\title{
¿Peligra la escultura del Peine del Viento por la fuerza del oleaje que lo azota? Diseño de un ABP orientado al aprendizaje activo en la formación de Arquitectos Técnicos Is there in danger the Comb of the Wind sculpture because of the surge"s force that flogs it? Design of a PBL orientated to the active learning in the formation of Technical Architects
}

\author{
Marieta, Cristina $a^{a}$; Retegi, Aloña ${ }^{a}$; Leon, Iñigo ${ }^{b}$ \\ ${ }^{a}$ Dpto. de Ingeniería Química y del Medio Ambiente, ${ }^{b}$ Dpto. de Arquitectura \\ Escuela de Ingeniería de Gipuzkoa, Universidad del País Vasco (UPV/EHU), España \\ (cristina.marieta@ehu.eus)
}

\begin{abstract}
In this communication, the design of a PBL, Problem Based Learning, for the teaching of physical and mechanical properties of materials in the subject of Fundamentals of Materials I for a degree in Technical Arquitecture is presented. This new methodology of teaching orientated to the active learning was implemented in the academic course 2012/13. On the basis of the results obtained in the exams, we can confirm that the methodology is suitable and positive for the acquisition of the results of learning proposed for the subject.
\end{abstract}

Keywords: PBL, materials, properties

\section{Resumen}

En esta comunicación, se presenta el diseño de un $A B P$, aprendizaje basado en problemas, para la enseñanza de las propiedades físicas y mecánicas de los materiales en la asignatura de Fundamentos de Materiales I del grado de Arquitectura Técnica. Esta nueva metodología de enseñanza orientada al aprendizaje activo se implementó en el curso académico 2012/13, y en base a los resultados obtenidos en los exámenes parciales, se puede confirmar que la metodología resulta adecuada y positiva para la adquisición de los resultados de aprendizaje propuestos para la asignatura.

Palabras clave: $A B P$, materiales, propiedades 


\section{Introducción}

Las asignaturas de Fundamentos de Materiales I y Materiales II (Materiales de Construcción en la antigua titulación de Arquitectura Técnica) son asignaturas del primer y del segundo curso respectivamente, del Grado de Arquitectura Técnica de la Escuela de Ingeniería de Gipuzkoa.
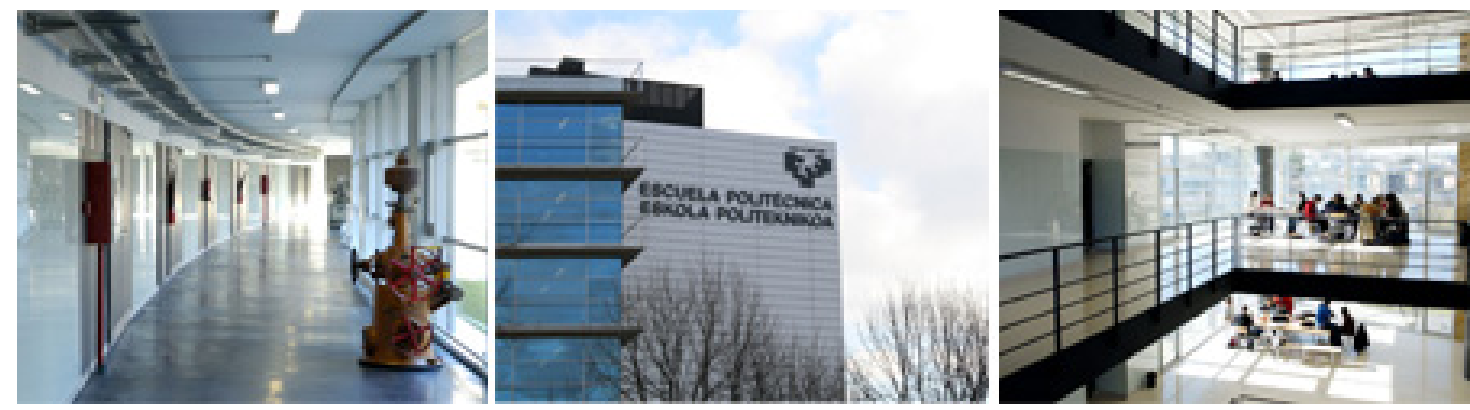

Fig. 1 Fotografías de la Escuela de Ingeniería de Gipuzkoa donde se imparte el Grado de Arquitectura Técnica

Ambas asignaturas corresponden al Módulo de Química y Geología de 18 créditos ECTS, son de carácter obligatorio, se imparten en castellano y en euskara, y una vez cursadas y aprobadas, el estudiante adquirirá los siguientes resultados de aprendizaje:

1. Conocerá la relación entre el origen y la aplicación de los materiales, y la nomenclatura científica y técnica para su actuación en un equipo multidisciplinar, y secundariamente, obtendrá formación inicial para una posterior especialización.

2. Relacionará los conceptos químicos con los minerales, rocas y materiales de origen artificial de uso más frecuente, así como conocerá las menas de los metales y otros materiales industriales.

3. Sistematizará los procesos, los minerales y las rocas correspondientes por su génesis, y no por un arbitrario nomenclátor comercial.

4. Conocerá la reactividad de los materiales de construcción frente a diferentes agentes externos y su relación con los potenciales procesos patológicos.

5. Se familiarizará con el trabajo en el laboratorio conducente a la identificación y análisis de los materiales o de sus componentes. Para ello ha de conocer la metodología de los ensayos de determinación de las características de los materiales de construcción.

6. Aplicará los conocimientos químicos en el impacto ambiental así cómo en el reciclado de los materiales de construcción, apoyando la gestión y el tratamiento de los residuos de las actividades constructivas.

Para que el estudiante llegue a alcanzar estos resultados de aprendizaje tiene que realizar una serie de actividades obligatorias, tales como las prácticas de laboratorio y defensa de un trabajo al finalizar la asignatura de Materiales II, y superar un examen de conocimientos teóricos y prácticos, lo cual requiere una importante dosis de trabajo tanto dentro como fuera del aula. Para que este trabajo sea productivo y se alcancen los objetivos propuestos, se requiere un mínimo de motivación. Sin embargo, desgraciadamente, la falta de motivación es una constante en la enseñanza universitaria, así como en general, en todos los niveles educativos. En el caso concreto del Grado de Arquitectura Técnica, se podría pensar que este desinterés se debe a la actual coyuntura económica, especialmente difícil en el sector de la construcción. No obstante, aunque si bien este puede ser un factor importante, no es único y determinante. Las actuales formas de ocio y aprendizaje fruto de las nuevas tecnologías, incrementan las capacidades cognitivas de nuestros estudiantes. La infinidad de estímulos 
externos que reciben y la amplitud de maneras de enfrentarse a los desafíos, hacen que las tradicionales metodologías de enseñanza basadas en clases magistrales y resolución de problemas teóricos les resulten aburridas y poco motivadoras, dando como resultado estudiantes pasivos que en algunos casos terminan por fracasar. Por todo esto, como primera aproximación a la búsqueda de nuevas metodologías de enseñanza más acordes a los tiempos que vivimos, hemos diseñado un ABP, aprendizaje basado en problemas, de aplicación en la asignatura de Fundamentos de Materiales I. En un ABP los estudiantes trabajan independientemente para "explorar" en un problema propuesto por el profesor. Dicho problema se plantea de manera que cuando el estudiante trabaje en su "resolución" se vaya encontrando con los objetivos curriculares de la titulación, actuando el profesor como facilitador de la tarea más que como fuente de conocimiento de la disciplina. La diferencia fundamental con los problemas teóricos tradicionales es que en el ABP el objetivo final no es la resolución del problema sino que es la discusión sobre la resolución del problema lo que lleva a la experiencia de aprendizaje del estudiante. Además, con este planteamiento pedagógico se pretende que los estudiantes adquieran, a parte de las competencias propias de la titulación, las siguientes competencias transversales:

1. Aprender de forma independiente.

2. Aplicar nuevos conocimientos en la resolución de distintos problemas similares a los que se les presentarán en el desempeño de distintas facetas de su trabajo.

3. Trabajar en equipo de forma supervisada.

4. Identificar sus objetivos, en este caso de aprendizaje.

5. Gestionar su tiempo de forma eficaz beneficiándose en este proceso de la colaboración de sus compañeros.

En la presente comunicación, se presenta el diseño de un ABP implementado en la asignatura de Fundamentos de Materiales I y se realiza la evaluación del ABP en base a los resultados académicos obtenidos por los alumnos.

\section{EI ABP, Aprendizaje Basado en Problemas}

Para la planificación de un ABP se deben realizar las siguientes acciones fundamentales:

1. Seleccionar los objetivos que enmarcados dentro de las competencias establecidas en la materia, se pretende que los estudiantes logren con la actividad.

2. Plantear la situación-problema sobre la que los estudiantes tendrán que trabajar, la cual debe ser:

a. Relevante para la práctica profesional de los estudiantes.

b. Compleja, pero no imposible, para que suponga un reto al estudiante.

c. Amplia para que los estudiantes puedan formularse preguntas y abordar la problemática con una visión de conjunto.

3. Orientar las reglas del trabajo en equipo y las actividades conducentes a la resolución del problema.

4. Establecer perfectamente los tiempos de las actividades.

5. Organizar sesiones de tutoría donde los estudiantes puedan consultar sus dudas, incertidumbres, logros... 


\subsection{Contexto del ABP dentro de la asignatura}

La asignatura de Fundamentos de Materiales I se divide en dos bloques temáticos: Fundamentos de materiales y Materiales de origen natural. A su vez, el primer bloque temático se divide en dos sub-bloques: introducción a la Ciencia de los Materiales y Propiedades de los Materiales, en el primer sub-bloque se describen conceptos básicos sobre los materiales y en el segundo se trata el estudio, análisis y cálculo de las propiedades de los materiales, concretamente se estudian las propiedades físicas y mecánicas, y es en este $40 \%$ de la asignatura donde se ha implementado el ABP.

\subsection{Objetivos pedagógicos}

- El estudiante será capaz de planificar tareas, asignar responsabilidades y coordinar el trabajo en equipo.

- El estudiante será capaz de plantear diferentes estrategias de resolución de problemas y elegir las más adecuadas.

- El estudiante será capaz de analizar resultados y argumentar en equipo y posteriormente transmitir la información oral y escrita utilizando la terminología específica del area y las herramientas informáticas apropiadas: Gráficos, Power Point, Videos, simulaciones...

- El estudiante será capaz de definir, determinar experimentalmente mediante ensayos normalizados y calcular, propiedades físicas y mecánicas de materiales de construcción.

- El estudiante será capaz de desarrollar habilidades propias del trabajo en el laboratorio, concretamente de las conducentes a la identificación y análisis de los materiales o de sus componentes.

\subsection{Situación-problema}

El escenario de la situación-problema del ABP se ubica en una construcción real: El Peine del Viento de Donostia-San Sebastián. Este es un conjunto escultórico que se encuentra en un extremo de la bahía de la Concha, formado por rocas naturales, estructuras metálicas y unas terrazas rocosas desde las que se puede observar la obra arquitectónica del escultor Eduardo Chillida. Se eligió esta construcción para el diseño del ABP debido a que integra los conceptos en cuanto a materiales y propiedades físicas a analizar según el temario de la asignatura. $A$ partir de la pregunta: ¿Peligra la escultura del peine del viento por la fuerza del oleaje que lo azota? Se genera la situación-problema o problema estructurante que sirve de hilo conductor para el desarrollo del ABP.

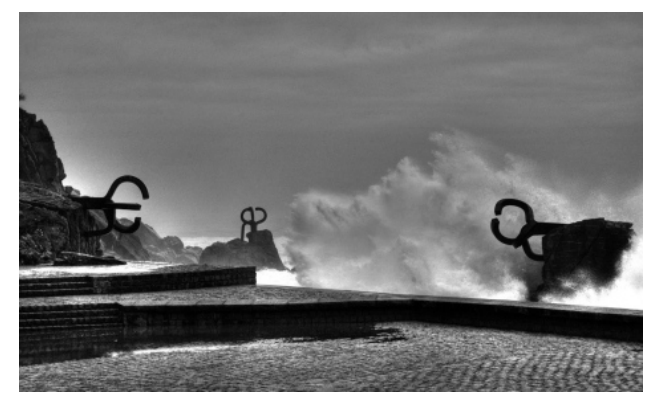

Fig. 2 Fotografía del oleaje impactando contra el Peine del Viento 


\subsection{Actividades en el desarrollo del ABP}

Para facilitar el desarrollo del proceso de enseñanza-aprendizaje y orientar al estudiante, el problema estructurante se aborda mediante tres sub-problemas:

A. Primer sub-problema: Se trabajan las propiedades físicas de los materiales. Para ello se analizan las propiedades de las rocas donde se anclan las esculturas: volúmenes, densidades, porosidades, humedad...

B. Segundo sub-problema: Se trabajan las propiedades mecánicas de los materiales. En este caso se han elegido las esculturas de acero ancladas sobre las rocas para su estudio debido a que a partir de las propiedades mecánicas de los metales (en especial del acero) se pueden describir los ensayos mecánicos más importantes de los materiales de construcción, tales como tracción, flexión, compresión impacto, fatiga...

C. Tercer sub-problema: Se trabajan las propiedades acústicas y térmicas de los materiales de construcción.

En cada sub-problema se vuelve a plantear una situación-problema, basada en el escenario inicial, para cuya resolución se realizan diferentes actividades basadas en técnicas de aprendizaje cooperativo: pósters y puzles.

* Póster: Tipo de comunicación en la cual se utiliza un cartel grande, generalmente con una imagen o fotografía, que se cuelga en una pared y/o en un panel habilitado para ello. Posibilita la transmisión concisa, clara y permanente de una información. En el presente caso se utilizará la pizarra donde dividida en sectores, cada grupo con varias palabras o una frase corta, defenderá sus conclusiones.

** Puzle: Se hacen grupos de tres personas y a cada persona se le asigna un problema. Individualmente cada componente trabaja el problema. Posteriormente, hay una reunión de expertos, es decir, los miembros de cada grupo con el mismo problema se reúnen para comentar sus conclusiones. Se vuelve a reunir el grupo inicial y tiene lugar una puesta en común. Finalmente se presentan los resultados obtenidos.

\subsubsection{Primer sub-problema: propiedades físicas de los materiales}

¿Puede el Peine del Viento estar sufriendo cambios debido al oleaje?

En la tabla 1 se muestra a modo de ejemplo una de las cinco actividades propuestas para el desarrollo de este sub-problema.

Tabla 1 Descripción de la actividad 1-3

\begin{tabular}{|c|c|}
\hline \multicolumn{2}{|c|}{ ACTIVIDAD 1-3: Propiedades físicas fundamentales: pesos, volúmenes y densidades } \\
\hline Modo de trabajo & Grupos de 3 alumnos propuestos por el profesor \\
\hline Tiempo estimado & 90 min (presencial) \\
\hline Evaluación & Cuestiones a resolver + ejercicio \\
\hline Recursos & Apuntes de la asignatura en Moodle. \\
& Conocimientos previos del alumno \\
Discusión en grupo de las cuestiones propuestas. Exposición póster de la tabla por cada grupo. Discusión \\
\hline
\end{tabular}


entre grupos si hubiera diferencias en algún aspecto. Preguntas del profesor por si algún grupo no ha detectado las diferencias entre algunas propiedades físicas. La actividad finaliza con una puesta en común de los ejercicios propuestos

\subsubsection{Segundo sub-problema: propiedades mecánicas de los materiales}

¿Resistirá el Peine del Viento los embates del tiempo?

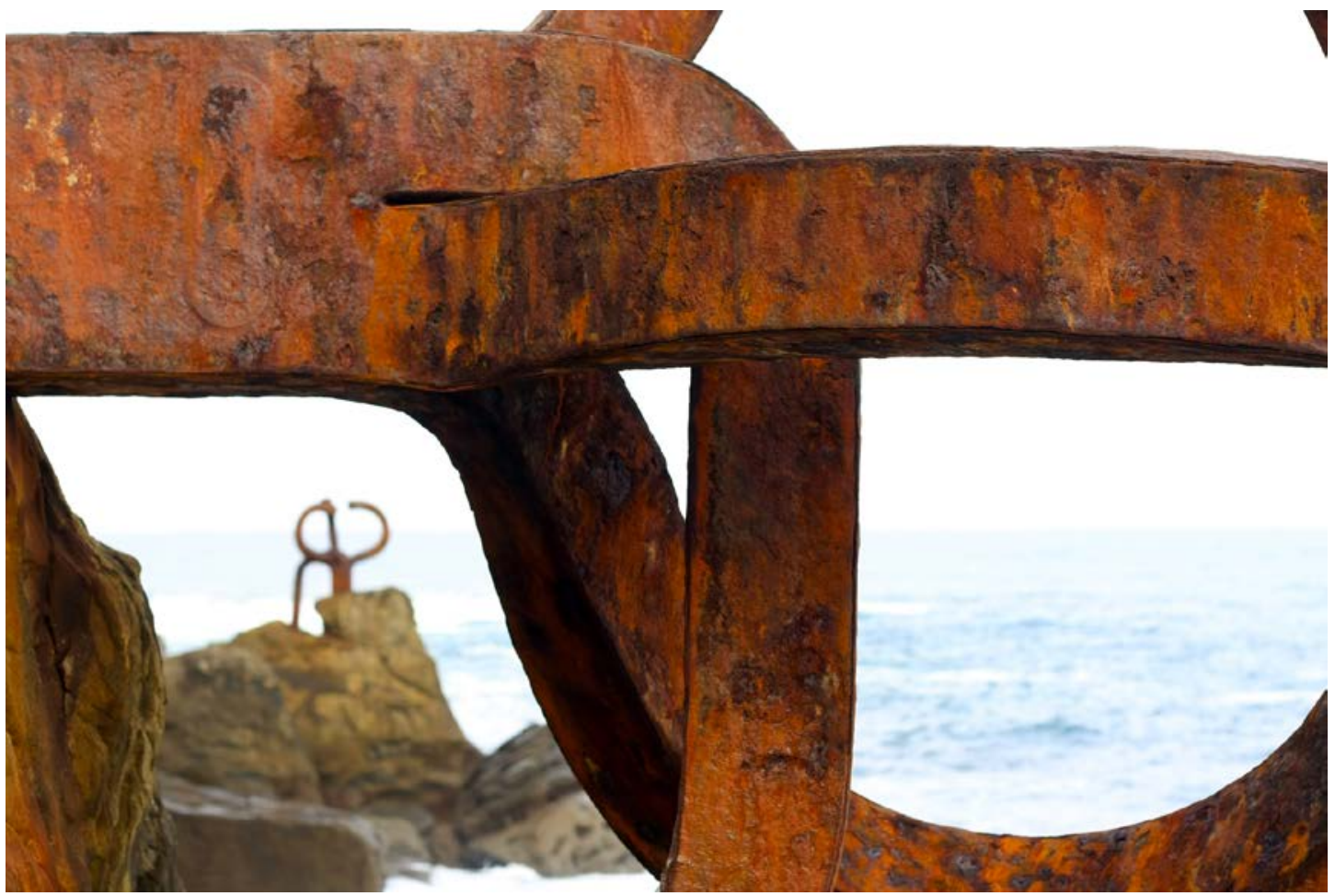

Fig. 3 Fotografía de la tercera escultura del Peine del Viento y detalle de la segunda

En la tabla 2 se muestra la primera de las cinco actividades propuestas para el desarrollo de este sub-problema.

Tabla 2 Descripción de la actividad 2-1

\begin{tabular}{|c|c|}
\hline $\begin{array}{r}\text { ACTIVIDAD 2-1: Propiedades mecánicas: Resistencia mecánica, elasticidad, } \\
\text { rigidez, límite elástico, ductilidad, dureza y tenacidad } \\
\text { trabajo }\end{array}$ & $\begin{array}{r}\text { Puzle (3 personas, 3 documentos: } \\
\text { Documento 1, Documento 2, Documento 3) (Se muestra em la figura : } \\
\text { Act. 2.1.1: Búsqueda personal (no presencial) } \\
\text { Act. 2.1:2: Reunión de grupo en aula } \\
\text { Act. 2.1.3: Reunión de expertos en aula } \\
\text { Act. 2.1.4: Reunión de grupo para resolución de cuestiones en aula } \\
\text { Corrección discusión en clase y resolución de problemas prácticos }\end{array}$ \\
\hline
\end{tabular}




\begin{tabular}{|c|c|}
\hline $\begin{array}{c}\text { Tiempo } \\
\text { estimado }\end{array}$ & $\begin{array}{c}6 \text { horas presenciales: } \\
\text { Act. 2.1.1: } \mathrm{np}^{\star} \\
\text { Act. 2.1:2: } \mathrm{p}(1 \mathrm{~h}) \\
\text { Act. 2.1.3: } \mathrm{p}(1 \mathrm{~h}) \\
\text { Act. 2.1.4: } \mathrm{p}(2 \mathrm{~h}) \\
\text { * } \mathrm{p} \text { : presencial, en aula } \\
\text { np: no presencial, fuera del aula } \\
\text { Corrección y discusión en clase: } \mathrm{p}(2 \mathrm{~h})\end{array}$ \\
\hline Recursos & $\begin{array}{c}-\quad \text { Anexo III (Puzle actividad 2.1) } \\
\text { - Se facilitará al alumno apuntes a través del moodle } \\
\text { Bibliografía: Introducción a la Ciencia e Ingeniería de los Materiales, William } \\
\text { D. Callister, Jr. Limusa-Wiley, México. 2009; Fundamentos de la Ciencia e } \\
\text { Ingeniería de Materiales, William F. Smith, McGraw-Hill, México. 2006. }\end{array}$ \\
\hline Evaluación & Cuestiones a resolver, auto-evaluación en moodle, examen \\
\hline \multicolumn{2}{|c|}{$\begin{array}{l}\text { Trabajo personal fuera de aula y puzle en equipo en clase. La actividad finaliza con una puesta } \\
\text { en común de los ejercicios propuestos. Autoevaluación personal del alumno mediante resolución } \\
\text { de problemas colgados en moodle con sus resultados. Examen }\end{array}$} \\
\hline
\end{tabular}

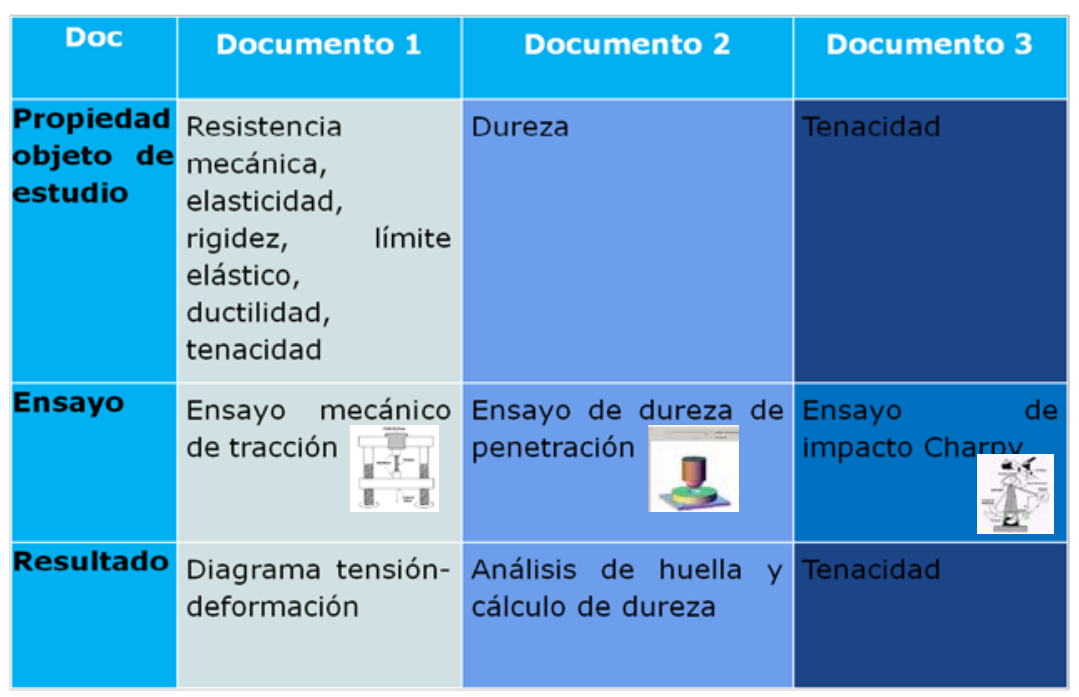

Fig. 4 Esquema del contenido del puzle de la actividad 2-1

\subsubsection{Tercer sub-problema: propiedades acústicas y térmicas}

¿Es admisible la intensidad sonora registrada en las viviendas próximas al peine del viento? Según la normativa en vigor, ¿supera los umbrales de contaminación acústica? Además, si te encargaran la reparación de la fachada, ¿qué material elegirías para asegurar el confort de los vecinos en sus viviendas teniendo en cuenta la climatología de la zona? 


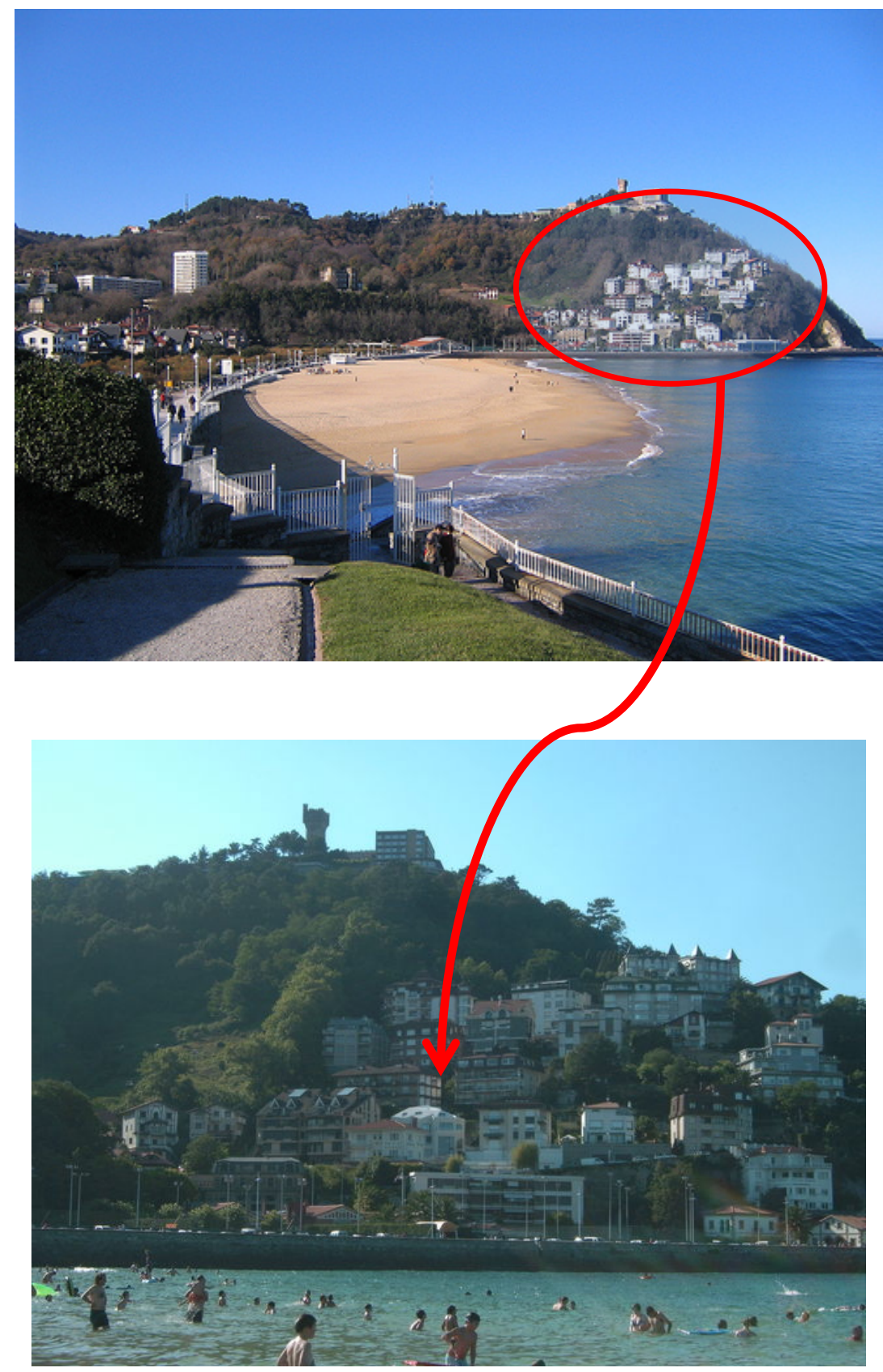

Fig. 5 Fotografías donde se ubican las viviendas objeto de estudio

En la tabla 3 se muestra la primera de las cinco actividades propuestas para el desarrollo de este sub-problema. 
Tabla 3 Descripción de la actividad 3-1

\begin{tabular}{|c|c|}
\hline \multicolumn{2}{|c|}{$\begin{array}{l}\text { ACTIVIDAD 3-1: Propiedades acústicas. Umbrales de contaminación acústica: cómo se mide, se } \\
\text { calcula y representación gráfica. }\end{array}$} \\
\hline Modo de trabajo & En grupos de 3 personas formados por los alumnos \\
\hline Tiempo estimado & 120 min (presencial) \\
\hline Evaluación & $\begin{array}{l}\text { Entregable y evaluable. Se valorará tanto el informe que entregue cada } \\
\text { grupo como la exposición oral que realicen. }\end{array}$ \\
\hline Recursos & $\begin{array}{l}\text {-Texto de la actividad -Normalmente los alumnos traen el portátil a clase y } \\
\text { en caso contrario utilizarán los disponibles en la universidad. }\end{array}$ \\
\hline \multicolumn{2}{|c|}{$\begin{array}{c}\text { En clase se procede a la lectura del texto que servirá de introducción a las propiedades acústicas de los } \\
\text { materiales (la lectura se realiza en grupo) ( } 2 \text { min). Después de } 5 \text { minutos de reflexión donde el grupo } \\
\text { discutirá sobre las palabras clave relacionadas con la contaminación acústica, deben escribir en la } \\
\text { pizarra } 3 \text { cuestiones/palabras que no hayan entendido sobre el texto. El profesor resolverá con la ayuda } \\
\text { de los demás grupos de trabajo las cuestiones más sencillas ( } 5 \text { min) y propondrá } 2 \text { aspectos a resolver } \\
\text { por los alumnos: }\end{array}$} \\
\hline & $\begin{array}{l}\text { 1- Representación gráfica de la escala acústica. Detectar umbral de } \\
\text { audición y umbral doloroso } \\
\text { 2- ¿Qué magnitud es la que se cuantifica con } 65 \text { dB? ¿Cuál es su } \\
\text { significado teórico? }\end{array}$ \\
\hline \multicolumn{2}{|c|}{$\begin{array}{l}\text { La mitad de los grupos (se mantiene los grupos de } 3 \text { iniciales) resolverá la cuestión a y la otra mitad de } \\
\text { los grupos la cuestión b ( } 20 \mathrm{~min} \text { ). Posteriormente se reunirán los grupos que han resuelto la cuestión a y } \\
\text { los grupos que han resuelto la cuestión b para discutir y llegar a una puesta en común (5 min). El } \\
\text { profesor elegirá al azar un alumno que haya resuelto la cuestión a y otro de la cuestión b para que } \\
\text { expliquen en clase mediante exposición oral ( } 5 \text { min). El profesor realizará preguntas que considere } \\
\text { oportunas si detecta que los alumnos no han identificado todos los objetivos de aprendizaje de la } \\
\text { actividad, orientando mediante propuestas (5-10 min). }\end{array}$} \\
\hline
\end{tabular}

\section{Resultados y discusión}

A partir de la comparativa de los resultados de los exámenes parciales de propiedades físicas y de propiedades mecánicas obtenidos en el primer bloque de la asignatura en los cuatro últimos cursos se hace una valoración del ABP.

En las figuras 3 y 4 se presentan los porcentajes de estudiantes que obtuvieron las calificaciones de suspenso, aprobado, notable y sobresaliente en los cursos 2012/13, 2013/14, 2014/15 y 2015/16 en el examen de propiedades físicas de los materiales (figura 3 ) y en el examen de propiedades mecánicas (figura 4).

La barra en rojo corresponde al curso 2012/13 en el cual se utilizó la metodología de enseñanza tradicional, es decir, clases magistrales y problemas de aplicación teóricos. En el curso 2013/14 es cuando se implementó el ABP. En el caso de propiedades físicas, se puede ver como el aprendizaje, valorado en base a los resultados obtenidos en el examen, con el ABP es notablemente superior al obtenido con metodologías tradicionales, como se desprende de la importante disminución de suspensos. Con respecto a las propiedades mecánicas, si bien en los primeros cursos de implementación de la metodología el descenso no fue tan evidente como en el caso de las propiedades físicas, la tendencia es a disminuir la cantidad de suspensos. Con respecto al resto de calificaciones, en general, también se observa una tendencia a la mejora. 


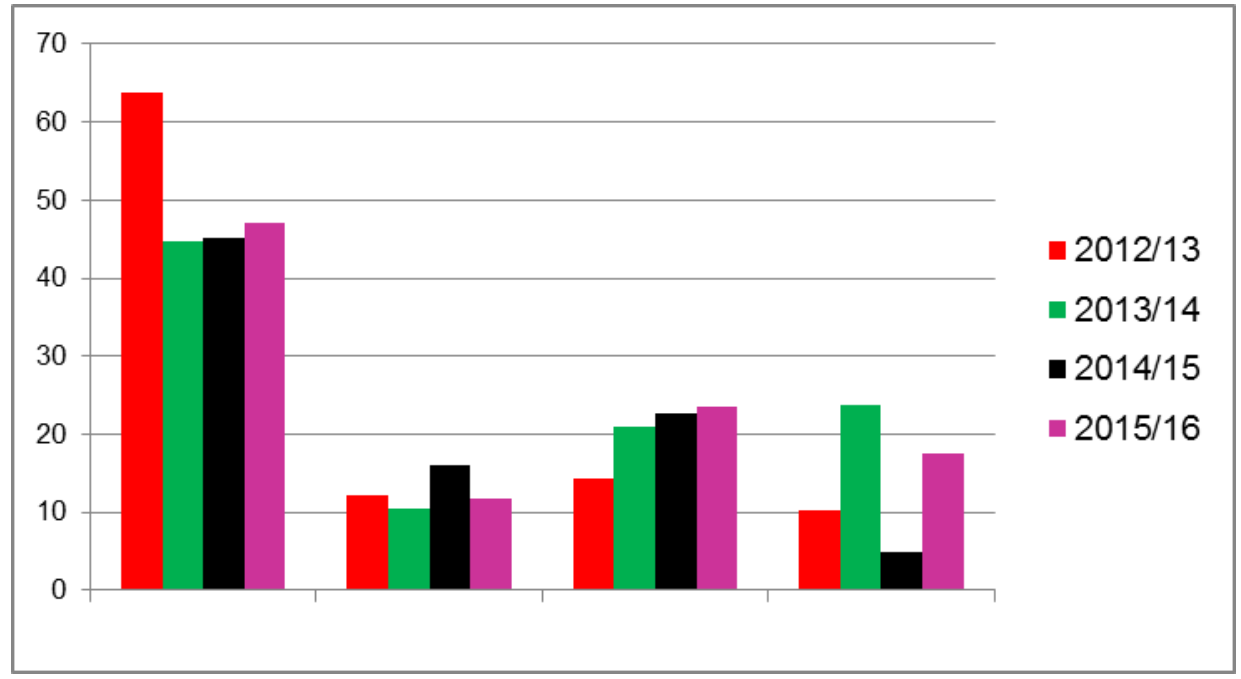

Suspenso Aprobado Notable Sobresaliente

Fig 3 Resultados académicos obtenidos por los estudiantes en la parte de propiedades físicas en el bloque temático Fundamentos de Materiales en el sub-bloque propiedades de los materiales, propiedades físicas

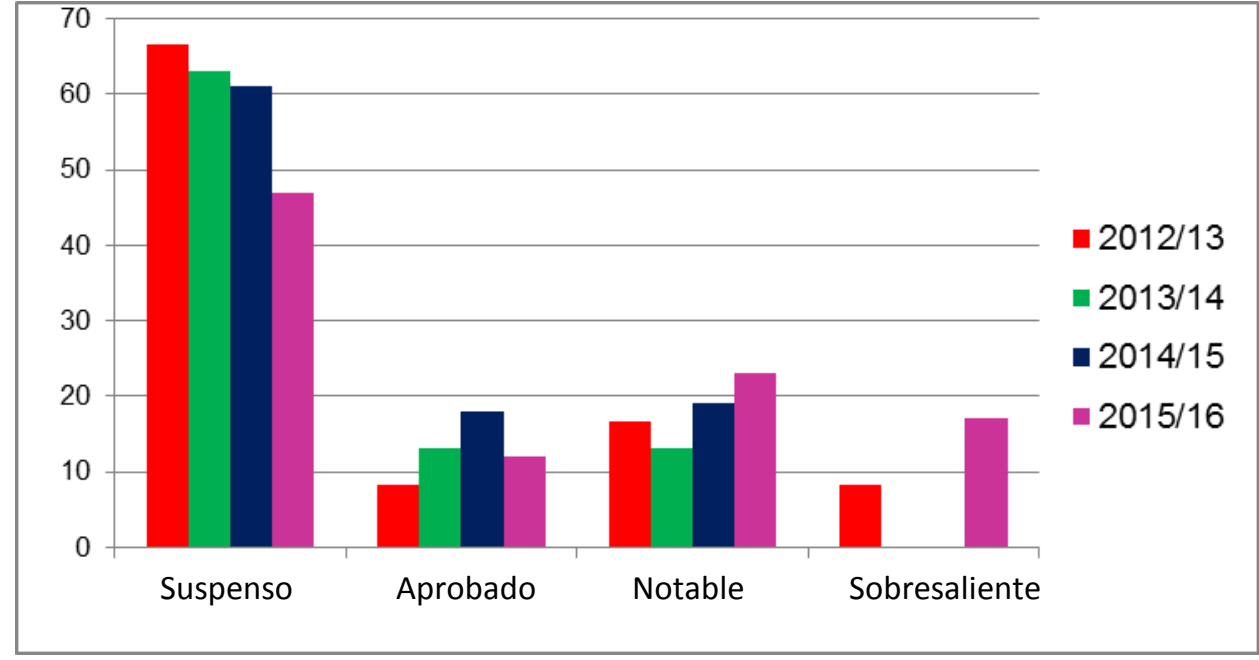

Fig 4 Resultados académicos obtenidos por los estudiantes en la parte de propiedades físicas en el bloque temático Fundamentos de Materiales en el sub-bloque propiedades de los materiales, propiedades mecánicas

\section{Conclusiones}

En vista de los resultados obtenidos, no cabe más que concluir que la implementación del ABP propuesto en el estudio de las propiedades físicas y mecánicas de los materiales ha sido positivo en el proceso de aprendizaje de los estudiantes de primer curso de materiales del grado de Arquitectura Técnica. Por lo tanto, estamos considerando la posibilidad de diseñar ABPs para el resto de los temas e incluso en colaboración con otras disciplinas del grado. 


\section{Agradecimientos}

Los autores agradecen al programa ERAGIN de formación del profesorado en metodologías activas de enseñanza de la UPV/EHU, por la ayuda proporcionada para la realización del presente trabajo y especialmente se agradece la ayuda de Estibaliz Saez de Camara.

\section{Referencias}

BARROWS, H. S. (1986). "A Taxonomy of problem-based learning methods. Medical" en Education, vol. 20, p. 481-486.

DE CASO FUERTES, A. M., GARCIA SANCHEZ, J. N., ARIAS GUNDIN, O., FIDALGO REDONDO, R. y FERNANDEZ MARTINEZ, M. (2006). "El aprendizaje basado en problemas: revisión de estudios empíricos internacionales" en Revista de educación, p. 397-418.

ESCRIBANO, A. y DEL VALLE, A. (2008). El aprendizaje basado en problemas. Una propuesta metodológica en Educación Superior. Madrid: Narcea.

HUBER, G. L. (2008). “Aprendizaje activo y metodologías educativas" en Revista de Educación, p. 59-91.

LLORENS MOLINA, J. A. (2010). "El aprendizaje basado en problemas como estrategia para el cambio metodológico en los trabajos de laboratorio" en Química Nova, p. 994-999.

MARLIES, B., DOCHY, F. y STRUYVEN, K. (2013). "The effects of different learning environments on students' motivation for learning and their achievement" en British Journal of Educational Psychology, p. 484-501.

TORRES GORDILLO, J. J. y PERERA RODRIGUEZ, V. H. (2010). "La rúbrica cómo instrumento pedagógico para la Tutorización y evaluación de los aprendizajes en el Foro online en educación superior" en Revista de Medios y Educación, p. 141-149.

Memorias ANECA, VERIFICA y Uniqual del Grado en Arquitectura Técnica. 